\title{
Often neglected: PLGA/PLA swelling orchestrates drug release - HME implants
}

\author{
C. Bode,${ }^{1}$ H. Kranz,${ }^{2}$ A. Fivez,${ }^{1}$ F. Siepmann, ${ }^{1}$ J. Siepmann ${ }^{1, *}$ \\ ${ }^{1}$ Univ. Lille, Inserm, CHU Lille, U1008, F-59000 Lille, France \\ ${ }^{2}$ Bayer AG, Muellerstraße 178, 13353 Berlin, Germany \\ *correspondence: \\ Prof. Juergen Siepmann \\ University of Lille, College of Pharmacy, Inserm U1008 \\ 3 rue du Professeur Laguesse, 59006 Lille, France \\ juergen.siepmann@univ-lille.fr
}

\begin{abstract}
:
Different types of poly(lactic-co-glycolic acid) (PLGA)- and poly(lactic acid) (PLA)-based implants for controlled dexamethasone release were prepared by hot melt extrusion (HME). The lactic acid:glycolic acid ratio was varied (50:50, 75:25, 100:0) as well as the drug loading (1-15\%). Resomer RG 502H, RG 752H and R 202H (all with - $\mathrm{COOH}$ end groups) were studied. The implants were characterized before and after exposure to phosphate buffer $\mathrm{pH} 7.4$ at $37^{\circ} \mathrm{C}$. Interestingly, in all cases polymer swelling seems to play an "orchestrating" role for drug release: At early time points, the amounts of water penetrating into the systems are limited (since the macromolecules are hydrophobic and highly entangled). Consequently, only small amounts of drug can dissolve and the dissolved drug molecules are not sufficiently mobile to diffuse out to a noteworthy extent (negligible dexamethasone release for up to 6 weeks). However, the water that is able to enter the implants at early time points cleaves the polyesters right from the beginning. Due to the newly generated - $\mathrm{COOH}$ end groups and decreased chain length, the macromolecules become more and more hydrophilic and less entangled. In addition, water-soluble polymer degradation products build up a steadily increasing osmotic pressure, attracting water into the system. Once a critical polymer molecular weight threshold range (around $8 \mathrm{kDa}$ ) is reached, substantial implant swelling starts: The systems' volume increases up to 600-1700\% and the water contents exceeds 80$90 \%$ (partially approaching $100 \%$ ). Under these fundamentally altered conditions, significant drug amounts can dissolve and the dissolved drug molecules are sufficiently mobile to diffuse out of the implants: Drug release sets on. In brief, polymer swelling "orchestrates" the involved mass transport phenomena: It enables drug release after a certain lag time by fundamentally changing the conditions for drug dissolution and diffusion. Note that in other types of implants, additional mass transport phenomena might be involved, e.g. no burst release was observed from the investigated, initially non-porous implants.
\end{abstract}

Key words: PLGA; PLA; swelling; implant; drug release mechanism; diffusion; dissolution. 


\section{Introduction}

Poly(lactic-co-glycolic acid) (PLGA) and poly(lactic acid) (PLA) are commonly used matrix formers for parenteral controlled drug delivery systems [1-5], offering the following major advantages: (i) They are biodegradable [6-9]. Thus, there is no need to remove empty remnants after drug exhaust. (ii) They are biocompatible, even with brain tissue [10]. (iii) They can control the resulting drug release rates during various time periods [11-15], ranging from a few days up to several months [16-20]. Different types of dosage forms can be prepared, such as implants and microparticles [21-26]. Various products are commercially available since many years.

The basic idea is to trap the drug within a PLGA or PLA matrix to avoid immediate release of the entire drug dose upon administration. Different types of mass transport mechanisms are involved in the subsequent control of drug release [27-30]. This includes water penetration into the systems, polymer chain cleavage, drug dissolution and diffusion, as well as system erosion [31-35]. Depending on the dosage form dimensions and composition, also autocatalytic effects might play a major role [36-39]: Water rapidly penetrates into the entire system and degrades the polymer throughout the device ("bulk erosion") [40]. The final degradation products are short chain acids, which are water-soluble and diffuse out into the surrounding environment (due to concentration gradients). However, the diffusional mass transport rate can be much lower than the rate at which the short chain acids are generated due to polymer chain cleavage. Hence, the micro $\mathrm{pH}$ within the dosage form might locally drop [41-43], in particular at the center of the system (where the diffusion pathways are the longest). Since ester hydrolysis is catalyzed by acids, such local drops in micro $\mathrm{pH}$ can lead to accelerated PLGA/PLA degradation $[44,45]$.

Furthermore, in the case of initially porous system surfaces, drug release through these pores might be rapid at early time points, causing a "burst effect" [46]. But polymer swelling can close these pores and drug release can subsequently slow down [47] (because the mobility of a dissolved drug molecule is higher in a water-filled pore than in a PLGA network). In addition, polymer swelling has also been identified as the root cause for the onset of the final rapid drug release phase from different types of PLGA-based microparticles [48-50]. Often, the latter exhibit tri-phasic drug release patterns: An initial rapid release phase ("burst release") is followed by a phase with an about constant drug release rate (this rate is often low, sometimes even close to zero), and a final, again rapid drug release phase (leading to complete drug exhaust). Monitoring the swelling and drug release behavior of "single microparticles", it could be shown that the onset of substantial microparticle swelling (after a certain lag time) coincided with the onset of the final rapid drug release phase. Also, Friess and Schlapp [51] reported that "The process of swelling, erosion, and structural collapse identified by SEM corresponded to the time course of water uptake by the microparticles and the GM release profiles.", studying gentamicin (GM)-loaded PLGA microparticles. Berkland et al. [52] investigated monodisperse, fluorescein-dextran- and sulforhodamine B-labeled bovine serum albumin-loaded PLGA-based microparticles. They found that " $[i] n$ general, however, the transition [into the final rapid drug release phase] appears to some extent to be caused by the swelling of the particles and the generation of a thin, porous, polymer shell with a hollow core." Furthermore, Mylonaki et al. [53] reported that the final rapid release phase from atorvastatin-loaded, PLGA-based microparticles coincided with system swelling (the latter enhancing diffusion-driven drug release). However, yet the role of potential substantial polymer swelling for the control of drug release from PLGA/PLA-based implants is not fully understood. The onset of a final rapid drug release phase from PLGA-based implants prepared by compression has for example been attributed to potential osmotic effects ("Eventually, the osmotic pressure within the compact may build up to such an extent that it ruptured the compact") [54]. 
The aim of this study was to prepare different types of implants by hot melt extrusion (HME) and to characterize the systems thoroughly before and after exposure to the release medium (phosphate buffer $\mathrm{pH} 7.4,37^{\circ} \mathrm{C}$ ). The lactic acid:glycolic acid ratio was varied $(50: 50,75: 25,100: 0)$ as well as the type of monomer (PLGA, PLA). All the investigated polymers were amorphous [55]. Dexamethasone was chosen as drug, since it does not act as a plasticizer for PLGA [50], and is neither acidic nor basic (and does, thus, not accelerate polyester hydrolysis). The idea was to limit the complexity of the underlying drug release mechanisms. Based on these experimental results the role of polymer swelling was to be better understood for the control of drug release from PLGA/PLA-based implants. Please note that in this study specific types of implants were investigated and caution needs to be taken when drawing general conclusions. In particular, in this work:

(i) The implants were prepared by hot melt extrusion. Other types of preparation techniques can lead to different inner implant structures, e.g. an important pore network present from the beginning can potentially result in burst effects.

(ii) The drug was likely homogeneously distributed throughout the implants.

(iii) Three specific types of PLGA/PLA were studied: Resomer RG 502H, Resomer RG $752 \mathrm{H}$ and Resomer R 202H. Other types of PLGA/PLA exhibit different key properties, which can affect the relative importance of the involved mass transport phenomena and their kinetics.

(iv) The drug loading was varied from $1-15 \%$ (below the percolation threshold value) (" $1 \%$ drug loading" means $1 \mathrm{~g}$ implant contained $0.01 \mathrm{~g}$ drug).

(v) The drug was dexamethasone, exhibiting low aqueous solubility. Thus, important osmotic effects due to dissolved drug are unlikely.

Variations in the above listed items (and others) can be expected to potentially affect the importance of the underlying physico-chemical phenomena. Thus, the hypotheses described in this work should be viewed with some caution when considering other types of implants. For example, in certain implants, PLGA/PLA swelling might control only the later parts of drug release (e.g., similarly to polymer swelling controlling the onset of the final, rapid drug release phase in PLGA microparticles [48-50]).

\section{Materials and methods}

\subsection{Materials}

Poly(D,L-lactic-co-glycolic acid) (PLGA) (Resomer RG 502H: 50:50 lactic acid:glycolic acid, -COOH end groups; and Resomer $R G$ 752H: 75:25 lactic acid:glycolic acid, -COOH end groups) and poly(D,L-lactic acid) (PLA) (Resomer $R$ 202H: -COOH end groups) (Evonik, Darmstadt, Germany); dexamethasone (Discovery Fine Chemicals, Dorset, UK); acetonitrile and tetrahydrofuran (Fisher Scientific, Illkirch, France); ethanol $96 \%$ (VWR, Fontenay-sousBois, France). 


\subsection{Implant preparation}

Appropriate amounts of polymer (PLGA or PLA) and drug (dexamethasone) were mixed for $5 \mathrm{~min}$ at $98 \mathrm{rpm}$ in a Turbula Shaker-Mixer (T2A, Willy A. Bachofen, Basel, Switzerland), followed by $5 \mathrm{~min}$ manual blending in a mortar with a pestle. The mixtures were filled into $5 \mathrm{~mL}$ syringes (Injekt Luer Lock Solo, B Braun, Melsungen, Germany), equipped with a shortened $(1.5 \mathrm{~cm})$ 16G needle. Figure 1 shows schematically the

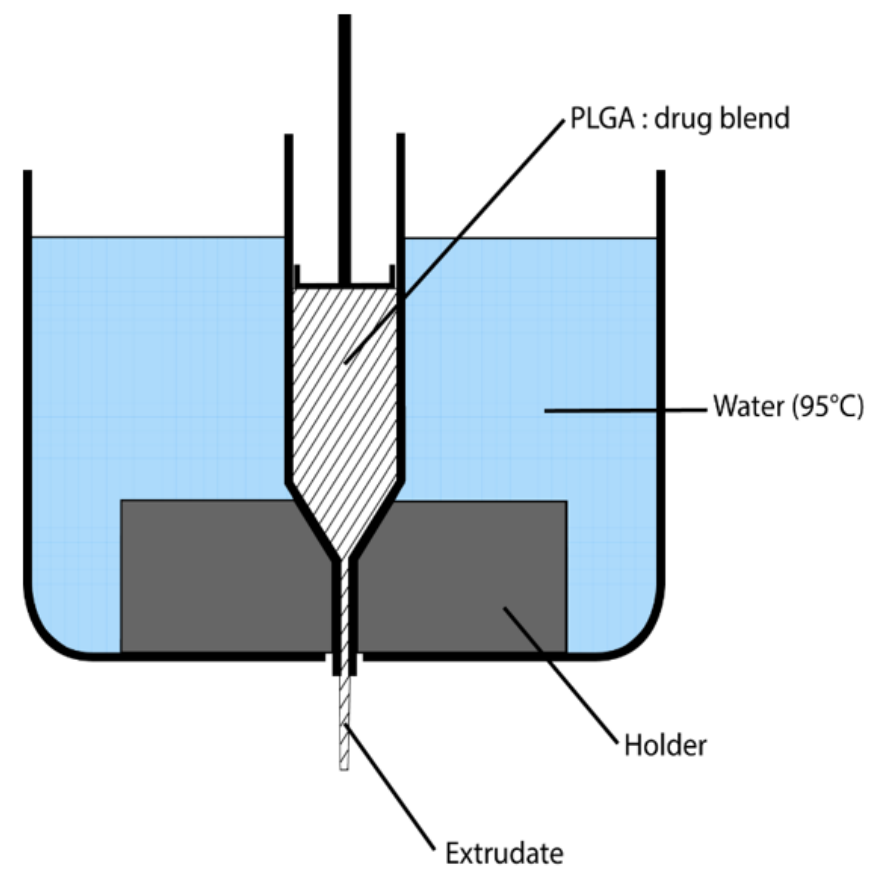

dexamethasone implants by hot melt extrusion. experimental set-up used to prepare the implants by hot melt extrusion. Briefly, a syringe was fixed in a holder. The water was kept at $95{ }^{\circ} \mathrm{C}$. After $5 \mathrm{~min}$, the content of the syringe was molten, and a texture analyzer (TAXT plus, Stable Micro Systems, Surrey, UK), equipped with a $50 \mathrm{~kg}$ load cell, was used to drive the syringe plunger downwards at a speed of $0.6 \mathrm{~mm} / \mathrm{min}$. The obtained extrudates were manually cut into cylinders (5 $\mathrm{mm}$ length), using a heated blade.

Figure 1: $\quad$ Schematic presentation of the experimental set-up used to prepare

\subsection{Implant characterization}

The diameter of the implants was measured with a SMZ-U microscope (Nikon, Tokyo, Japan), equipped with an AxioCam ICc1 camera and the Axiovision Zeiss Software (Carl Zeiss, Jena Germany).

The practical drug loading was determined as follows: Samples were dissolved in $5 \mathrm{~mL}$ of a 1:4 (v/v) ethanol:acetonitrile mixture. The drug content of the solutions was analyzed using a Thermo Fisher Scientific Ultimate 3000 Series HPLC, equipped with a LPG 3400 SD/RS pump, an auto sampler (WPS-3000 SL) and a UV-Vis detector (VWD-3400RS) (Thermo Fisher Scientific, Waltham, USA). Samples were centrifuged for $2.5 \mathrm{~min}$ at $10,000 \mathrm{rpm}$ (Centrifuge Universal 320; Hettich, Tuttlingen, Germany) and filtered with a $0.45 \mu \mathrm{m}$ PVDF syringe filter (Millex-HV, Merck Millipore, Tullagreen, Ireland). Ten $\mu \mathrm{L}$ samples were injected into an A C18 RP column (Gemini $3 \mu \mathrm{m} \mathrm{C18} 110 \AA$ A, 100 mm x $4.6 \mathrm{~mm}$; Phenomenex, Le Pecq, France). The mobile phase consisted of a 33:67 (v/v) acetonitrile:water mixture, the flow rate was $1.5 \mathrm{~mL} / \mathrm{min}$. Dexamethasone had a retention time of approximately $3.8 \mathrm{~min}$, the detection wavelength was $\lambda=254 \mathrm{~nm}$. The calibration curve was linear ( $\mathrm{R}>$ 0.999 ) within the range of 0.06 to $0.00003 \mathrm{mg} / \mathrm{mL}$. All experiments were conducted in triplicate. Mean values $+/$ - standard deviations are reported.

In vitro drug release: Implants were placed into Eppendorf vials (1 implant per vial), filled with $4 \mathrm{~mL}$ phosphate buffer $\mathrm{pH} 7.4$ (United States Pharmacopeia $40,37^{\circ} \mathrm{C}$ ) and horizontally shaken at $80 \mathrm{rpm}$ and $37^{\circ} \mathrm{C}$ (GFL 3033, Gesellschaft fuer Labortechnik, Burgwedel, Germany). At pre-determined time points, the release medium was completely replaced. The amount of dexamethasone in the withdrawn bulk fluid was determined by HPLC-UV analysis, 
as described above (injection volume: $100 \mu \mathrm{L}$ ). If implant remnants remained at the end of the observation period, the amount of potentially "not released" dexamethasone was determined as follows: The remnants were freeze-dried for $3 \mathrm{~d}$ (conditions: Table S1; Christ Epsilon 2-4 LSC; Martin Christ, Osterode, Germany) and the lyophilisates were dissolved in a 4:1 (v/v) acetonitrile:ethanol mixture. The solutions were filtered $(0.45 \mu \mathrm{m}$ PVDF filter syringes) and analyzed for their drug contents by HPLC-UV (as described above). Note that these experiments showed that in case of incomplete drug release at the end of the observation period, the amounts that had not been released, were experimentally recovered in the implant remnants (100\% mass balance). All experiments were conducted in triplicate. Mean values $+/$ - standard deviations are reported. In addition, the $\mathrm{pH}$ of the release medium was measured at pre-determined time points using a $\mathrm{pH}$ meter (InoLab $\mathrm{pH}$ Level 1; WTW, Weilheim, Germany) ( $\mathrm{n}=3$, mean values $+/$ - standard deviations are reported).

Implant swelling and erosion: Implants were treated as for drug release studies. At predetermined time points, specimen were withdrawn, excess water carefully removed using Kimtech precision wipes (Kimberly-Clark, Rouen, France) and weighed [wet mass ( $t)$ ]. The samples were lyophilized for $3 \mathrm{~d}$ (please see above) and weighed again [dry mass $(t)]$. The wet mass (\%) (t), water content $(\%)(t)$, and dry mass loss $(\%)(t)$ were calculated as follows:

$$
\begin{gathered}
\text { wet mass }(\%)(t)=\frac{\text { wet mass }(t)}{\text { initial weight }} \times 100 \% \\
\text { water content }(\%)(t)=\frac{\text { wet mass }(t)-\text { dry mass }(t)}{\text { wet mass }(t)} \times 100 \% \\
\text { drymass loss }(\%)(t)=\frac{\text { initial weight }- \text { dry mass }(t)}{\text { initial weight }} \times 100 \%
\end{gathered}
$$

where initial weight is the weight of the implants before exposure to the release medium $(t=$ $0)$. All experiments were conducted in triplicate. Mean values $+/-$ standard deviations are reported.

Polymer degradation: Implants were treated as for drug release studies. At pre-determined time points, specimen were withdrawn, freeze-dried for $3 \mathrm{~d}$ (please see above) and the lyophilisates were dissolved in tetrahydrofuran (at a concentration of $1.5 \mathrm{mg} / \mathrm{mL}$ ). The average polymer molecular weight (Mw) of the PLGA and PLA in the samples was determined by Gel Permeation Chromatography (GPC, Separation Modules e2695 and e2695D, 2419 RI Detector, Empower GPC software; Waters, Guyancourt, France), using a PLGel $5 \mu \mathrm{m}$ MIXED-D column (kept at $35^{\circ} \mathrm{C}$ ), 7.5 × $300 \mathrm{~mm}$ (Agilent Technologies, Interchim, Montluçon, France). The injection volume was $50 \mu \mathrm{L}$. Tetrahydrofuran was the mobile phase (flow rate: $1 \mathrm{~mL} / \mathrm{min}$ ). Polystyrene standards with molecular weights between 1,090 and 70,950 Da (Polymer Labaratories, Varian, Les Ulis, France) were used to prepare the calibration curve. All experiments were conducted in triplicate. Mean values $+/-$ standard deviations are reported.

Implant morphology: Implants were treated as for drug release studies. At pre-determined time points, specimen were withdrawn and freeze-dried as described above. Pictures were taken with an optical image analysis system (Nikon SMZ-U), equipped with a Zeiss camera (AxioCam ICc1). In addition, SEM pictures of implants were made before exposure to the release medium with a JEOL Field Emission Scanning Electron Microscope JSM-7800F (Tokyo, Japan). Samples were fixed with a ribbon carbon double-sided adhesive on the 
sample holder and covered with a fine chrome layer using the Gatan Model 682 Precision Etching and Coating System (Pleasanton, CA, USA).

\subsection{Determination of drug solubility}

The solubility of dexamethasone (as received) in phosphate buffer $\mathrm{pH} 7.4$ at $37{ }^{\circ} \mathrm{C}$ was determined in agitated glass flasks. An excess amount of dexamethasone powder (approximately $30 \mathrm{mg}$ ) was exposed to $80 \mathrm{~mL}$ bulk fluid, and kept at $37{ }^{\circ} \mathrm{C}$ under horizontal shaking (80 rpm; GFL 3033). At pre-determined time points, samples were withdrawn, immediately filtered $(0.45 \mu \mathrm{m}$ PVDF syringe filter), diluted and analyzed for their drug content by HPLC-UV analysis (as described above, using an injection volume of $20 \mu \mathrm{L}$ ). Measurements were performed until equilibrium was reached. Each experiment was conducted in triplicate. Mean values $+/$ - standard deviations are reported.

\section{Results and discussion}

Table 1 shows the practical drug loadings and initial dimensions of the investigated implants. All specimen were white, had a smooth surface and uniform appearance. Pictures of representative examples are shown in the top row of Figure 2.

Table 1: Dimensions and drug loadings of the investigated dexamethasone implants. Mean values $+/$ - standard deviations are indicated $(n=3)$.

\begin{tabular}{|c|c|c|c|c|c|c|c|}
\hline Polymer & & $1 \%$ & $2.5 \%$ & $5 \%$ & $7.5 \%$ & $10 \%$ & $15 \%$ \\
\hline \multirow[t]{3}{*}{ PLGA (RG 502H) } & Diameter $(\mathrm{mm})$ & $1.2 \pm 0.0$ & $1.4 \pm 0.0$ & $1.3 \pm 0.1$ & $1.2 \pm 0.1$ & $1.2 \pm 0.1$ & $1.2 \pm 0.1$ \\
\hline & Length (mm) & $5.8 \pm 0.0$ & $5.8 \pm 0.2$ & $5.9 \pm 0.2$ & $5.7 \pm 0.2$ & $5.8 \pm 0.4$ & $5.8 \pm 0.3$ \\
\hline & $\begin{array}{l}\text { Practical drug } \\
\text { loading (\%) }\end{array}$ & $0.8 \pm 0.0$ & $2.3 \pm 0.1$ & $4.7 \pm 0.1$ & $7.2 \pm 0.0$ & $9.1 \pm 0.0$ & $13.9 \pm 0.3$ \\
\hline \multirow[t]{3}{*}{ PLGA (RG 752H) } & Diameter $(\mathrm{mm})$ & $1.2 \pm 0.0$ & $1.1 \pm 0.0$ & $1.2 \pm 0.0$ & $1.4 \pm 0.0$ & $1.2 \pm 0.1$ & $1.1 \pm 0.0$ \\
\hline & Length (mm) & $5.4 \pm 0.2$ & $5.7 \pm 0.1$ & $5.3 \pm 0.6$ & $5.6 \pm 0.2$ & $5.7 \pm 0.2$ & $5.9 \pm 0.2$ \\
\hline & $\begin{array}{l}\text { Practical drug } \\
\text { loading (\%) }\end{array}$ & $0.9 \pm 0.1$ & $2.6 \pm 0.1$ & $4.7 \pm 0.0$ & $7.3 \pm 0.1$ & $9.9 \pm 0.3$ & $15.0 \pm 0.2$ \\
\hline \multirow[t]{3}{*}{ PLA (R 202H) } & Diameter $(\mathrm{mm})$ & $1.2 \pm 0.1$ & $1.3 \pm 0.1$ & $1.4 \pm 0.1$ & $1.3 \pm 0.1$ & $1.4 \pm 0.0$ & $1.3 \pm 0.1$ \\
\hline & Length (mm) & $5.8 \pm 0.1$ & $5.6 \pm 0.2$ & $5.7 \pm 0.0$ & $5.6 \pm 0.3$ & $5.6 \pm 0.3$ & $5.6 \pm 0.4$ \\
\hline & $\begin{array}{l}\text { Practical drug } \\
\text { loading (\%) }\end{array}$ & $1.0 \pm 0.1$ & $2.3 \pm 0.0$ & $4.8 \pm 0.3$ & $7.5 \pm 0.1$ & $9.7 \pm 0.1$ & $14.8 \pm 0.2$ \\
\hline
\end{tabular}


PLGA (RG 502H)

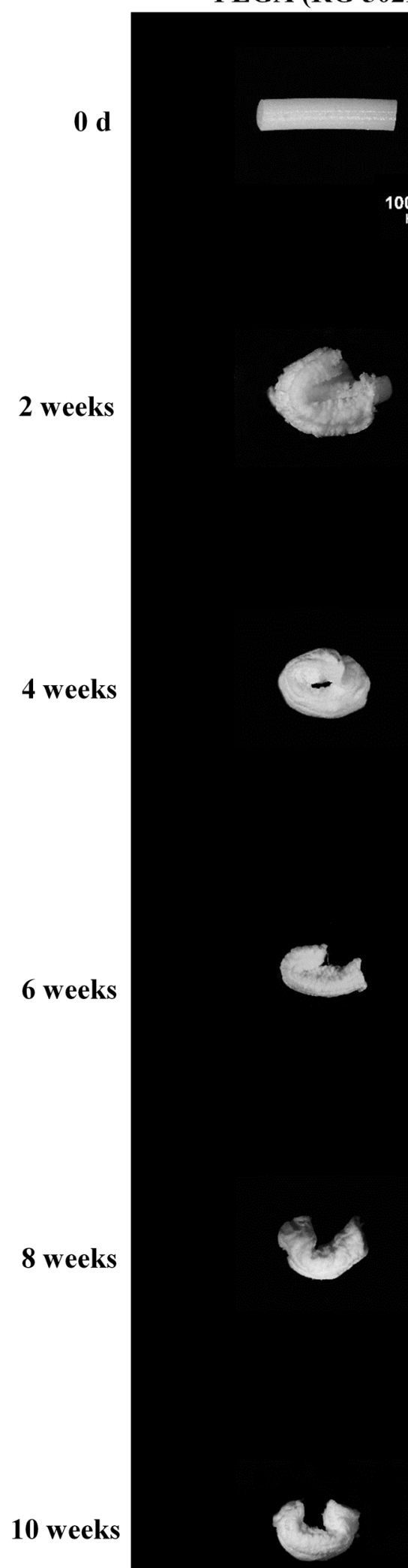

PLGA (RG 752H)

PLA (R 202H)

$000 \mu \mathrm{m}$
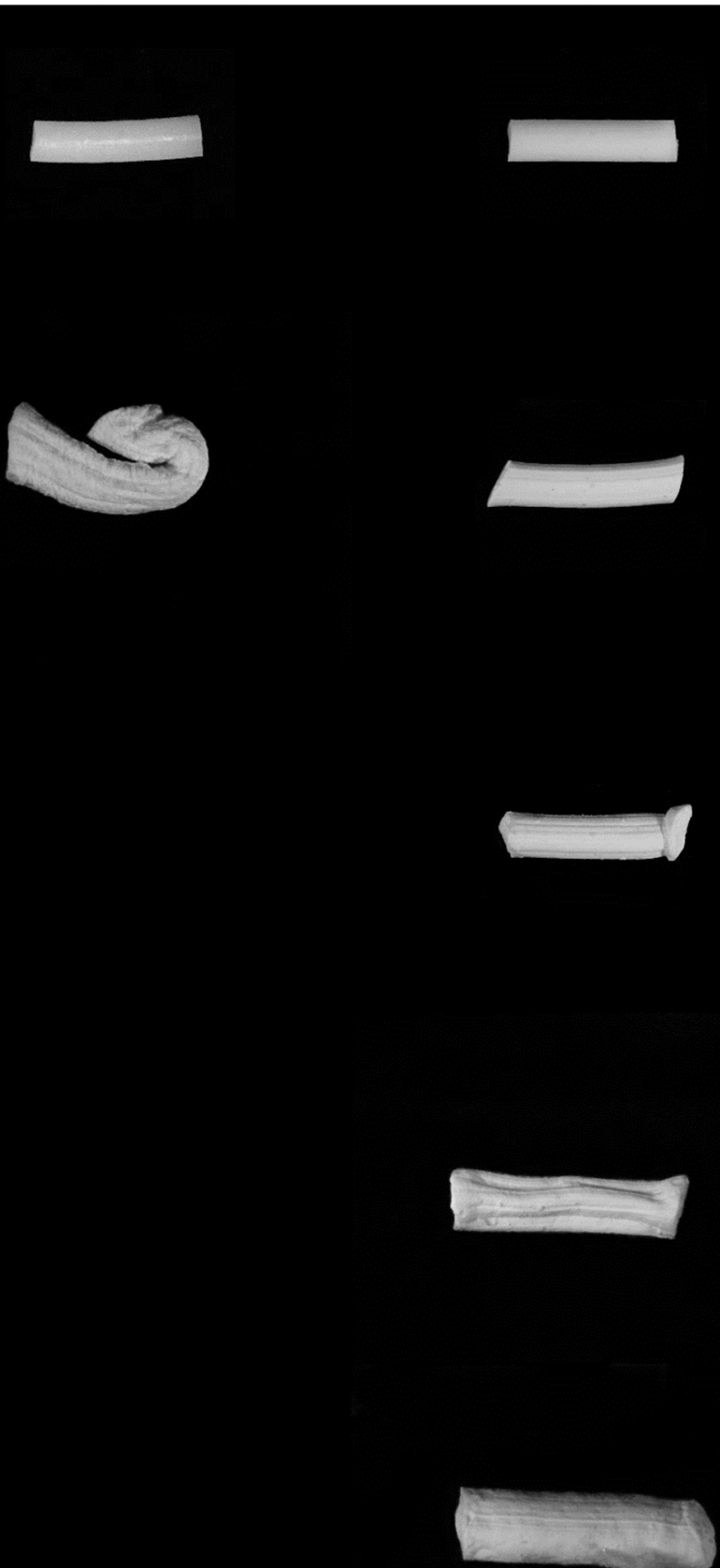

Figure 2: Macroscopic pictures of implants before and after exposure to phosphate buffer pH 7.4 (in the latter case after freeze drying). The implants contained $10 \%$ dexamethasone. In the case of PLGA RG 752H, the implants became too fragile to be handled after 4 weeks. 


\subsection{PLGA (RG 502H)-based implants}

Figure $3 \mathrm{a}$ illustrates the relative dexamethasone release rates from implants based on PLGA (RG 502H) (on the right hand side a zoom on the first 2 weeks is shown). The drug loading was varied from 1 to $15 \%$. As it can be seen, the relative release rate decreased with increasing initial drug content. This can be attributed to the difference in the $100 \%$ reference value for complete release, since the absolute drug release rates were similar prior to drug exhaustion for all drug loadings (Figure 3b): An increase in the drug amount corresponding to the $100 \%$ reference value leads to a decrease in the relative drug release rate, when the absolute release rate remains about constant.

Furthermore, during major time periods about constant drug release rates were observed. This might serve as an indication for the fact that limited drug solubility effects within the implants are of major importance, combined with perfect sink conditions in the surrounding bulk fluid. Upon water penetration into the systems, the drug is probably only partially dissolved, because the amounts of water within the implants are limited and since the solubility of dexamethasone in aqueous media is low (e.g. $77+/-4 \mu \mathrm{g} / \mathrm{mL}$ at $37{ }^{\circ} \mathrm{C}$ in phosphate buffer $\mathrm{pH}$ 7.4). Thus, dissolved and non-dissolved drug co-exist within the systems. Importantly, only dissolved drug is available for diffusion (non-dissolved drug cannot diffuse). Hence, irrespective of the initial drug content of the implants, saturated dexamethasone solutions likely exist within the systems. Released dissolved dexamethasone molecules are rapidly replaced by the partial dissolution of the non-dissolved drug excess. Combined with perfect sink conditions in the surrounding, well-stirred release medium, this results in about constant drug concentration differences in all cases (approximately "solubility - 0 ”). Furthermore, the initial implant dimensions are similar for all drug loadings (Table 1). In addition, since the polymer and drug are the same in all cases (and the drug loadings limited), also the drug permeability is likely similar. Thus, most of the key conditions for drug diffusion are similar, resulting in similar absolute drug diffusion rates $(=$ absolute drug release rates), as long as saturated drug solutions are provided within the implants (Figure 3b). Please note that different initial drug loadings can be expected to lead to different inner implant structures upon drug release. However, such differences do not seem to play an important role in the investigated ranges.

Once all non-dissolved drug excess within the implants is exhausted, released dissolved drug molecules are no more replaced and the dexamethasone concentration in the system decreases with time. This leads to decreasing drug concentration gradients (the driving forces for diffusion), and hence, to decreasing absolute and relative drug release rates (Figures $3 \mathrm{a}$ and b). Drug release "levels off", and complete drug release is achieved. With increasing initial drug content, this "drug exhaustion and leveling off" effect occurs at later time points.

Figure $3 c$ shows the dynamic changes in the implants' wet mass upon exposure to the release medium. Clearly, the wet mass substantially increased up to 1.5 weeks (irrespective of the drug loading), and then decreased again. The initial increase in wet mass is due to the penetration of water into the system, the subsequent decrease results from implant erosion and drug release. Interestingly, a lag time of about $4 \mathrm{~d}$ was observed for the onset of drug release (Figure 3a), irrespective of the initial drug loading. This corresponds to the time point from which on the water content of the implants substantially increased (Figure 3c). Hence, it seems that the drug "has to wait" for significant amounts of water to enter the system, before being released. Initially, dexamethasone is effectively trapped within the polymeric matrix. The amounts of water present within the system are not sufficient to allow for noteworthy drug dissolution and diffusion. Only once substantial amounts of water come in, important drug amounts can dissolve and diffuse out (the mobility of the polymer chains increases with increasing water content, resulting in increased drug mobility). This is consistent with the experimentally measured water contents of the implants upon exposure to the release medium (Figure 4a): After 1 week the implants consist of more than $80 \%$ water. Clearly, a drug can 
be expected to have a noteworthy mobility in a polymeric system with such a high water content. Please note that when looking into more detail, potentially "polymer-rich" and "water-rich" regions might be distinguishable in these systems, with different drug mobility. It would be interesting to investigate this aspect in future studies. 
PLGA (RG 502H)

a)

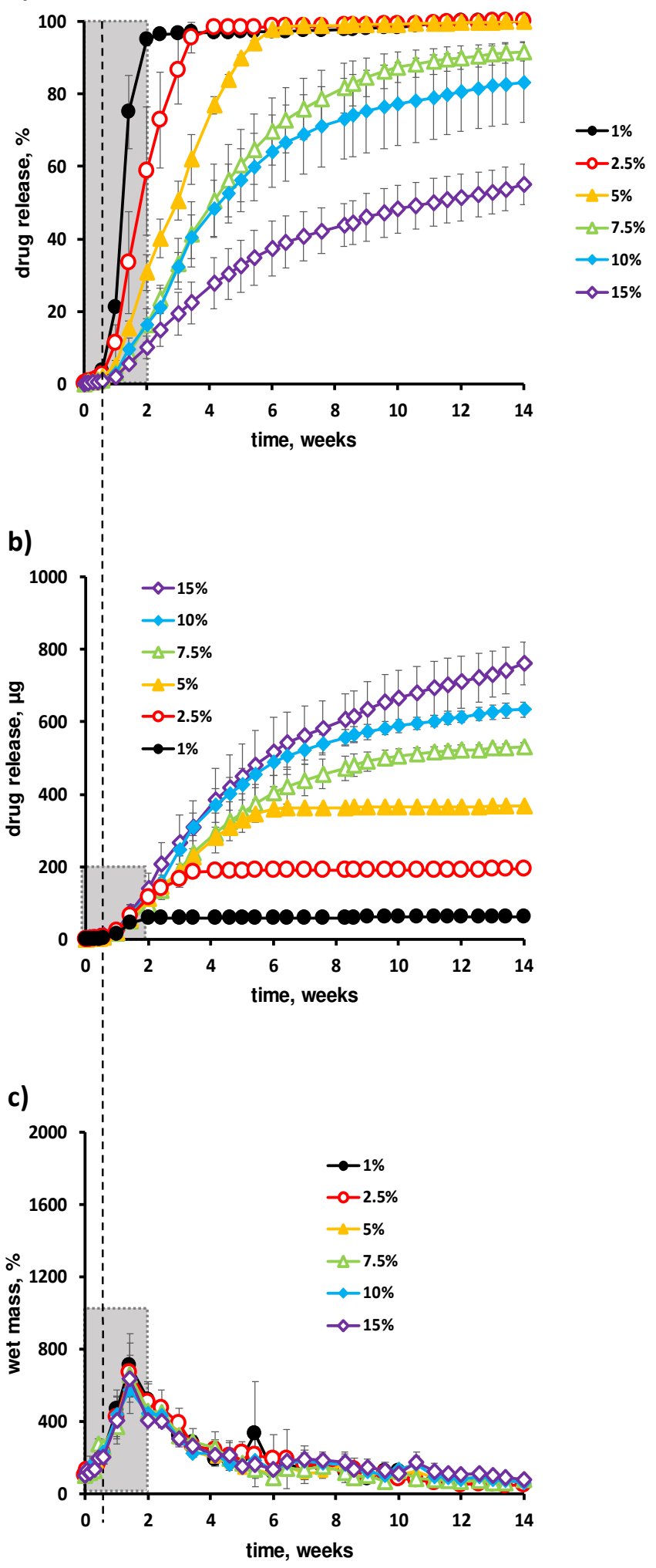

Zoom
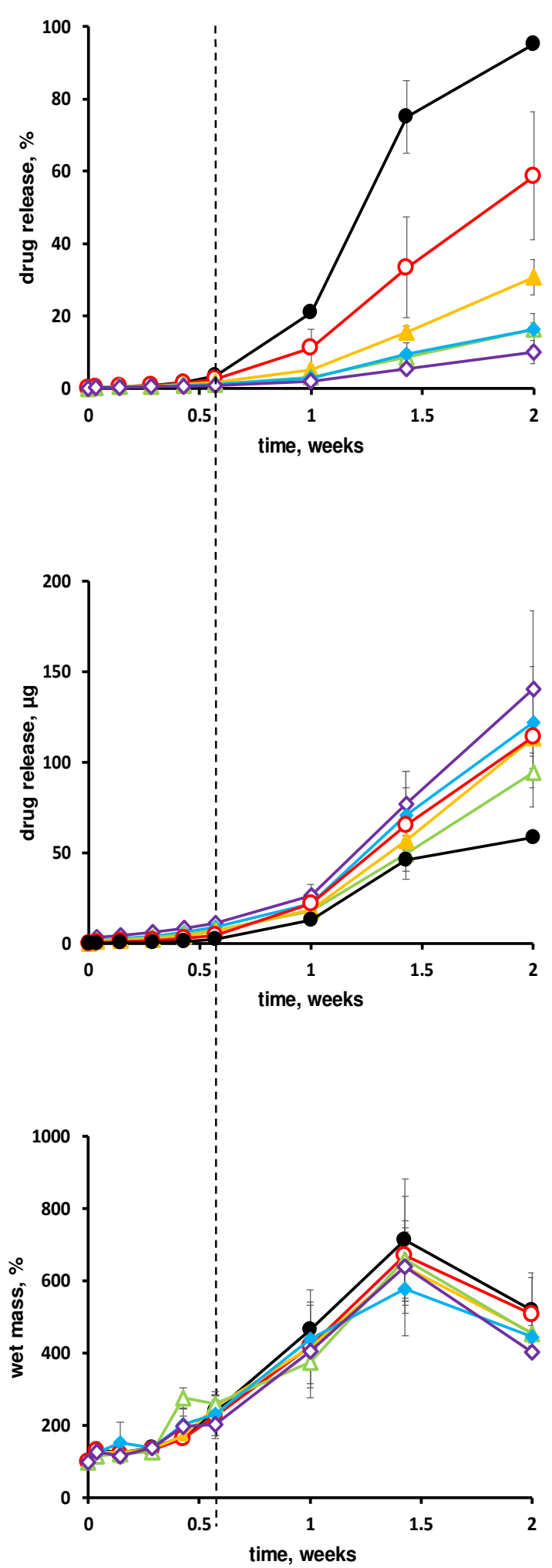

Figure 3: Impact of the initial drug loading of PLGA (RG 502H)-based implants (indicated in the diagrams) on the resulting: a) relative dexamethasone release kinetics, b) absolute dexamethasone release kinetics, and c) dynamic changes in the systems' wet mass after exposure to phosphate buffer $\mathrm{pH}$ 7.4. Mean values $+/$ - standard deviations are indicated $(\mathrm{n}=3)$. On the right hand side, zooms on early time points are shown (marked in grey on the left hand side). 
PLGA (RG 502H)

a)

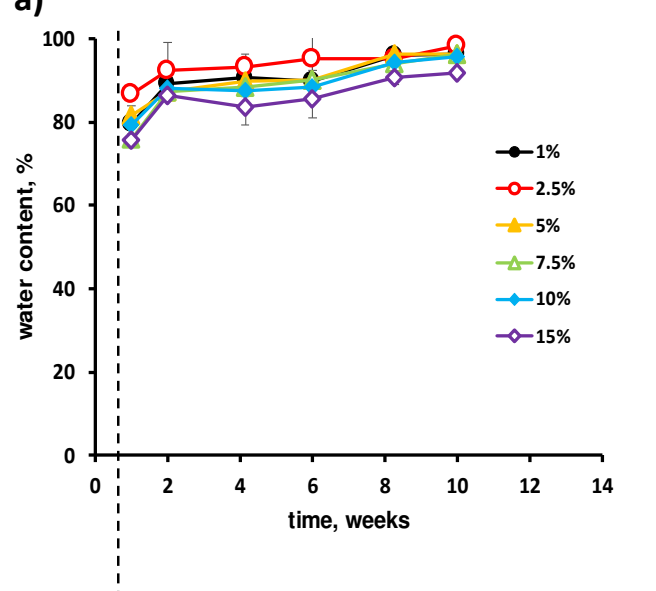

b)
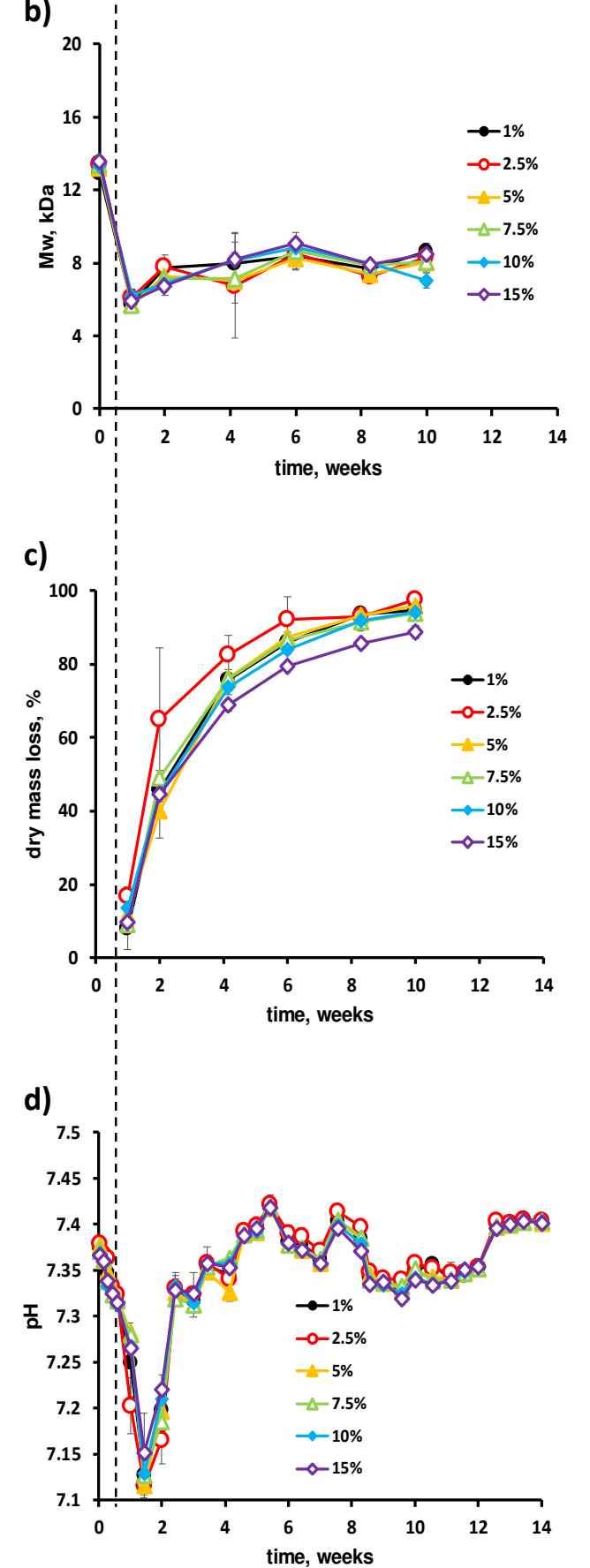

Figure $4 \mathrm{~b}$ shows the likely reason for this substantial increase in the water content of the PLGA (RG 502H)-based implants after about $4 \mathrm{~d}$ : The polymer molecular weight $(\mathrm{Mw})$ decreased from initially about $13-14 \mathrm{kDa}$ to about $8 \mathrm{kDa}$. Before exposure to the release medium, the polymer chains were rather long and, thus: (i) more hydrophobic, and (ii) more intensively mutually entangled. Note that (in brief) in the case of PLGA with - $\mathrm{COOH}$ end groups, the polymer backbone is hydrophobic and the end groups are hydrophilic. The limited amounts of water penetrating into the implants at early time points start cleaving the ester bonds (Figure 4b). The newly created $-\mathrm{COOH}$ groups render the macromolecular network more hydrophilic. In addition, the degree of polymer chain entanglement decreases. As soon as a critical threshold value range is reached (roughly around $8 \mathrm{kDa}$ ), the hydrophilicity of the PLGA chains becomes sufficiently high to allow substantial amounts of water to enter the system. In addition, the degree of polymer chain entanglement has decreased to such an extent that the network becomes more easily "expandable" (which is also a pre-requisite for substantial swelling). Furthermore, the presence of water-soluble degradation products can be expected to build up a steadily increasing osmotic pressure within the implants, attracting water into the system [27]. The consequence is tremendous implant swelling, e.g. the wet mass increased up to 600-800\% (Figure 3c). Note that since the chain length is not $100 \%$ uniform, it is preferable to consider a threshold value "range" rather than a single threshold "value". Also, please note that the indicated values are average polymer molecular weights in dynamically changing systems (e.g., water-soluble degradation products continuously diffuse out, while longer polymer chains are cleaved).

The experimentally measured dry mass loss kinetics and dynamic changes in the $\mathrm{pH}$ of the surrounding bulk fluid are in good agreement with these hypotheses: The substantial decrease in polymer molecular weight is accompanied by the release of water-soluble, short chain acids into the release medium, leading to a clear drop in the $\mathrm{pH}$ of the bulk fluid after 4-7 d (Figure 4d). Furthermore, this time point corresponds to the onset of substantial dry mass loss of the implants (Figure 4c). (Note that the increase in $\mathrm{pH}$ at later time points is due to the complete renewal of the phosphate buffer at each sampling time point.)

Figure 4: Impact of the initial drug loading of PLGA 
(RG 502H)-based implants (indicated in the diagrams) on the dynamic changes in the: a) water content, b) polymer molecular weight, c) dry mass of the systems upon exposure

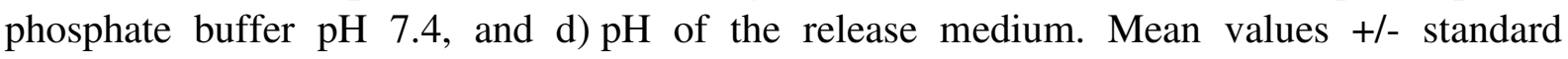
deviations are indicated $(n=3)$. 


\subsection{PLGA (RG 752H)-based implants}

Figures $5 \mathrm{a}$ and $\mathrm{b}$ show the relative and absolute dexamethasone release rates from PLGA (RG 752H)-based implants. In this case, the lactic acid:glycolic acid ratio was 75:25 (compared to 50:50 in the previous section). Importantly, the same phenomena were observed as in the case of PLGA (RG 502H)-based implants (essentially only the onset of the different events was shifted to later time points):

- During the first $10 \mathrm{~d}$ the relative and absolute release rates were close to zero, because the drug was effectively trapped within the polymeric systems: The water contents were limited at this stage (Figure 5d), resulting in low polymer permeability and limited amounts of water available for drug dissolution (only dissolved drug being able to diffuse). Note that the type of preparation technique (hot melt extrusion) resulted in nonporous implant surfaces (please see SEM pictures in Figure S1). Thus, water and drug transport through pores/cracks with direct surface access was negligible.

- However, the limited amounts of water that could penetrate into the implants caused polymer degradation right from the beginning (Figure 5e).

- After about $10 \mathrm{~d}$, the polymer molecular weight decreased to the critical polymer molecular weight threshold range around $8 \mathrm{kDa}$ (Figure 5e): This rendered the PLGA sufficiently hydrophilic to allow for substantial system swelling: Important amounts of water came in (Figure 5d). For example, the implants contained $85 \%$ water after 2 weeks. Thus, the drug could dissolve and became mobile within the polymeric systems: This caused the onset of dexamethasone release.

- Also, short chain acids (which were generated upon PLGA degradation) dissolved in the incoming water and became sufficiently mobile at this time point to diffuse out of the system (due to concentration gradients), into the surrounding bulk fluid: Consequently, the $\mathrm{pH}$ of the release medium significantly decreased after $10 \mathrm{~d}$ (Figure $5 \mathrm{~g}$ ).

- Due to the loss of short chain degradation products and due to drug release, the dry mass loss of the implants set on (Figure 5f).

- Interestingly, these phenomena were virtually independent of the initial drug content (only the $100 \%$ reference value for complete drug release was different). This can at least in part be explained by the fact that dexamethasone is not acting as a plasticizer for PLGA [50], nor is it an acidic or a base (and, thus, does not accelerate or cause ester hydrolysis). Furthermore, the drug loading was relatively limited (1-15\%).

The fact that most of these phenomena set on at later time points compared to PLGA (RG 502H)-based implants can at least partially be explained by the facts that: (i) the initial polymer molecular weight was higher (16 vs. $13 \mathrm{kDa}$ ), (ii) PLGA (RG 752H) contains more lactic-acid units than PLGA (RG 502H): This slows down the ester bond cleavage (due to sterical hindrance) and renders the polymer more hydrophobic (thus, it is more "difficult" for the water to come in). Consequently, the critical polymer molecular weight range around $8 \mathrm{kDa}$ is reached at a later time point (Figure 5e vs. Figure $4 \mathrm{~b}$ ), and the onset of system swelling is delayed.

Note that the water content of the implants approached $100 \%$ after 4 weeks (Figure 5d). Hence, drug mobility can be expected to substantially increase. This explains why the remarkable increase in system dimensions (volume increase up to $1700 \%$ : Figure 5c; pictures in Figure 2) did not significantly slow down drug release: The increase in the mobility of dissolved dexamethasone molecules can be expected to "roughly" compensate the increase in the lengths of the diffusion pathways. It would be interesting to study this aspect in more detail in the future. 
PLGA (RG 752H)

a)

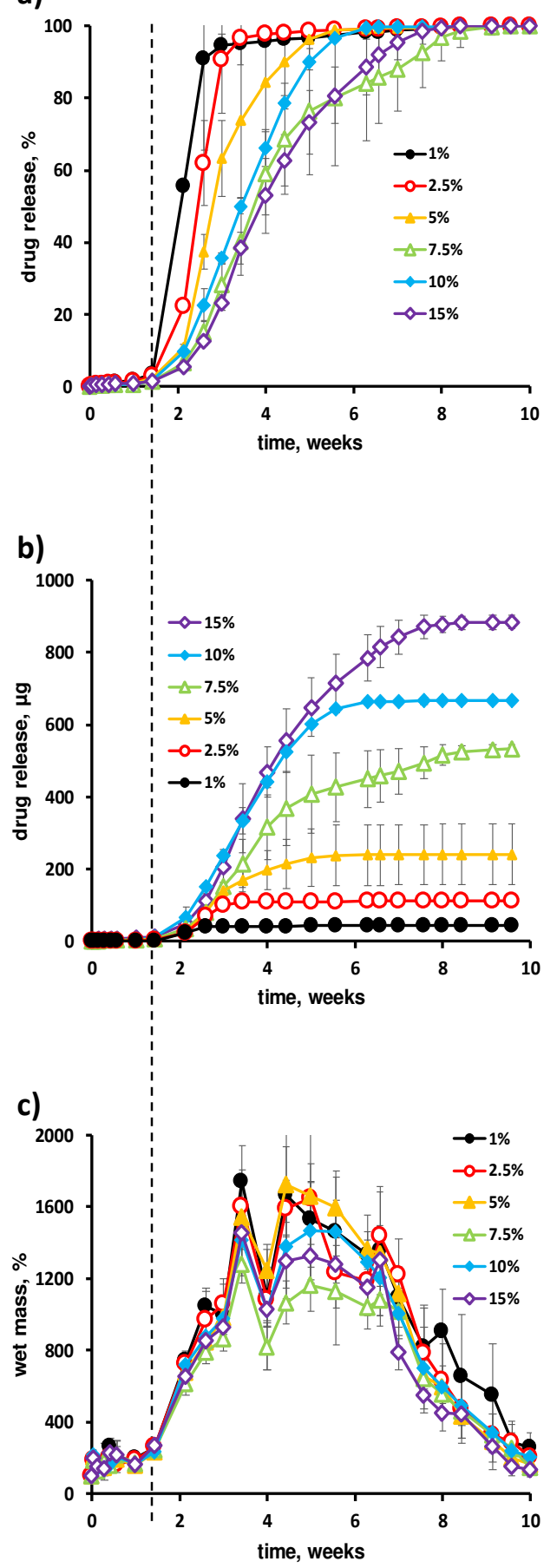

Figure 5: Impact of the initial drug loading of PLGA (RG 752H)-based implants (indicated in the diagrams) on the resulting: a) relative dexamethasone release kinetics, b) absolute dexamethasone release kinetics, c) changes in the systems' wet mass, d) water content, e) polymer molecular weight, f) dry mass upon exposure phosphate buffer $\mathrm{pH} \mathrm{7.4,} \mathrm{and} \mathrm{g)} \mathrm{pH}$ of the release medium. Mean values $+/$ - standard deviations are indicated $(n=3)$. d)

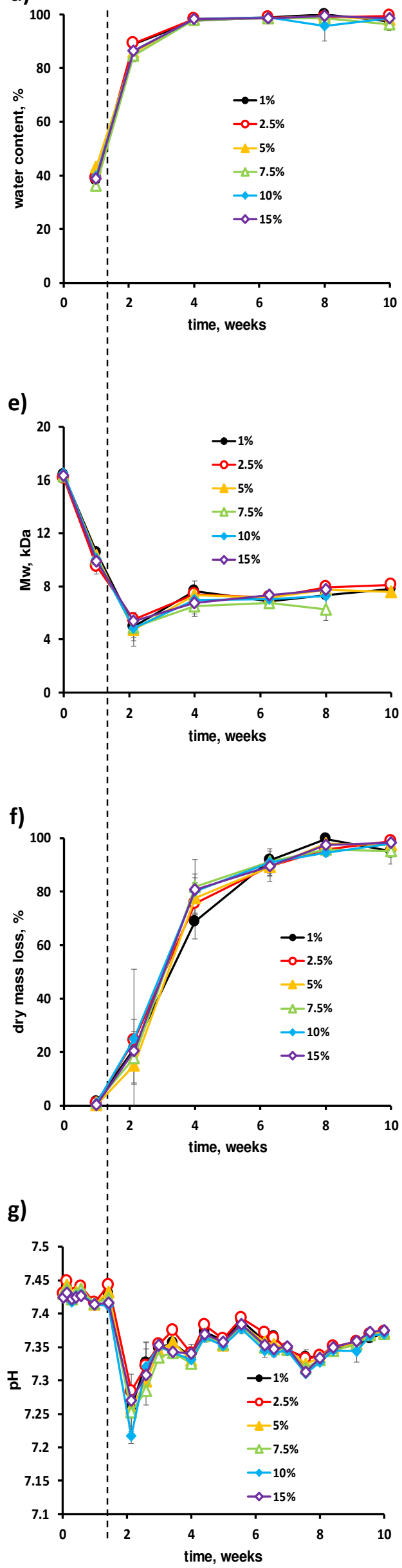




\subsection{PLA (R 202H)-based implants}

Figures $6 \mathrm{a}$ and $\mathrm{b}$ show the relative and absolute dexamethasone release rates from PLA (R 202H)-based implants, with an initial drug loading of 1 to $15 \%$. Again, a clear lag time was observed, coinciding with the lag time for implant swelling (Figure 6c). As with PLGA (RG 502H) and PLGA (RG 752H), also in the case of PLA (R 202H), the following phenomena occurred:

- Upon contact with the release medium, limited amounts of water penetrated into the implants, causing PLA degradation from the beginning (Figure 6e).

- As soon as the critical threshold range around $8 \mathrm{kDa}$ was reached (after about 6 weeks), substantial implant swelling set on (Figures 6e and c).

- The tremendous increase in the water content of the system (approaching $100 \%$, Figure 6d) allowed for drug dissolution and diffusion. Note that during the first 6 weeks, even up to $60 \%$ water content of the implants did not allow for noteworthy drug release (Figures 6a and d). This value is consistent with those observed with PLGA (RG 502H)and PLGA (RG 752H)-based implants (e.g., Figures 4a and 5d).

- In the highly swollen implants also the generated water-soluble short chain acids dissolved and were released into the surrounding bulk fluid, leading to a drop in the $\mathrm{pH}$ of the release medium (Figure 6g) and (together with drug release) to the onset of substantial dry mass loss of the implants (Figure 6f).

Again, no noteworthy impact of the drug loading was observed on the timing of these events. Interestingly, and in contrast to PLGA (RG 502H)- and PLGA (RG 752H)-based implants, the cylindrical geometry of the systems was kept during at least 8 weeks (Figure 2). This might at least partially be attributable to the slower polymer degradation (Figures $4 \mathrm{~b}, 5 \mathrm{e}$ and 6e): The longer the polymer chains, the more they are mutually entangled and the higher is the mechanical stability of the system. The observed deformation of the PLGA (RG 502H)and PLGA (RG 752H)-based implants during the experiments can be attributed to the fact that the implants became deformable and adapted their shape to the geometry of the bottoms of the Eppendorf vials (horizontally shaken at $80 \mathrm{rpm}$ ).

Furthermore, the onset time point for substantial polymer swelling was delayed compared to (RG 502H)- and PLGA (RG 752H)-based implants (Figures 3c, 5c and 6c). This can probably at least partially be explained by the fact that PLA $(\mathrm{R} 202 \mathrm{H})$ contains only lactic acid units, while PLGA contains lactic and glycolic acid units: The additional methyl group in the lactic acid units causes sterical hindrance for hydrolytic ester bond cleavage and renders the system more hydrophobic. Both effects slow down polymer degradation.

Comparing Figures 3a, 5a and 6a, it can be seen that the slope of the drug release curves (once dexamethasone release had started) increased in the following ranking order: PLGA $($ RG $502 \mathrm{H})<$ PLGA $(\mathrm{RG} \mathrm{752H)}<$ PLA $(\mathrm{R} \mathrm{202H})$. This is in good agreement with the observed dry mass loss rates of the implants (once dry mass loss had started), which increased in the same ranking order (Figures $4 \mathrm{c}, 5 \mathrm{f}$ and $6 \mathrm{f}$ ). The exact reasons for these differences are not yet fully understood. They are likely attributable to the differences in the polymeric structures (type of monomers and monomer ratio) [55]. Potential partial explanations might include the following: In the case of PLGA (RG 502H)-based implants, the resulting water contents even in the fully swollen states are lower compared to PLGA (RG 752H)- and PLA (R 202H)-based systems (Figures 4a, 5d and 6d). This leads to lower drug and short chain acid mobility. In the case of PLGA (RG 752H) and PLA (R 202H), the water contents in the fully swollen state are close to $100 \%$ (Figures $5 \mathrm{~d}$ and $6 \mathrm{~d}$ ). However, the pathways to be overcome are much longer in the case of PLGA (RG 752H)-based implants compared to PLA ( $\mathrm{R} 202 \mathrm{H}$ )-based systems, as evidenced by the very high wet mass values (Figure 5c vs. 6c). Please note that PLGA (RG 752H)-based implants became too fragile to be handled after 4 weeks. This is why no pictures could be taken (Figure 2), but they increased substantially in dimensions (visual observation). Longer pathways lead to slower release rates. 
a)

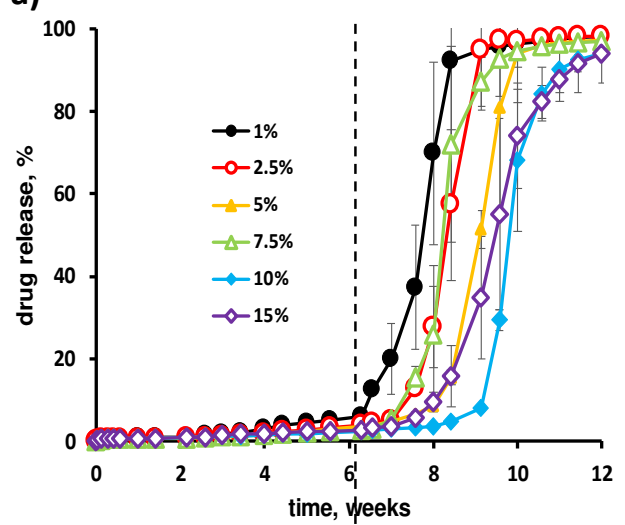

b)

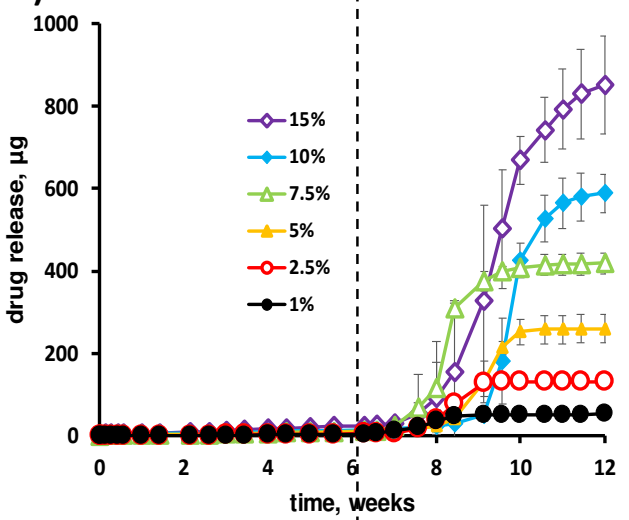

c)

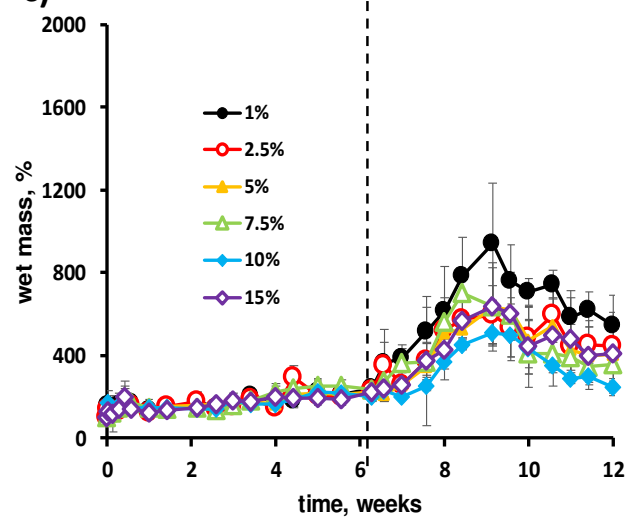

Figure 6: Impact of the initial drug loading of PLA (R 202H)-based implants (indicated in the diagrams) on the resulting: a) relative dexamethasone release kinetics, b) absolute dexamethasone release kinetics, c) changes in the systems' wet mass, d) water content, e) polymer molecular weight, f) dry mass upon exposure phosphate buffer $\mathrm{pH} 7.4$, and g) $\mathrm{pH}$ of the release medium. Mean values $+/$ - standard deviations are indicated $(n=3)$.
PLA (R 202H)

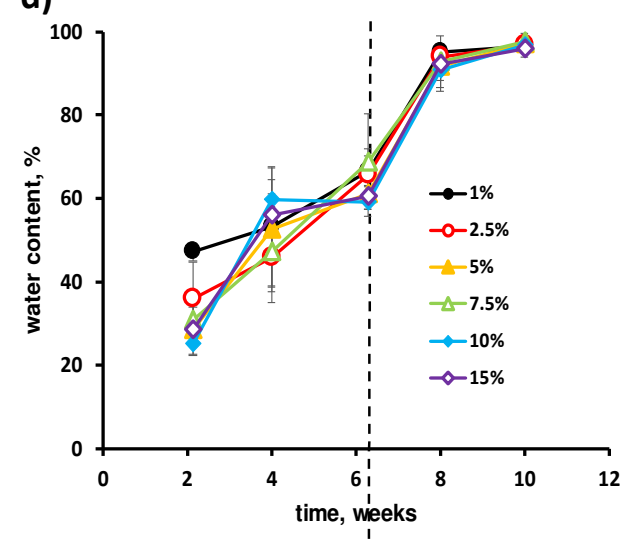

e)

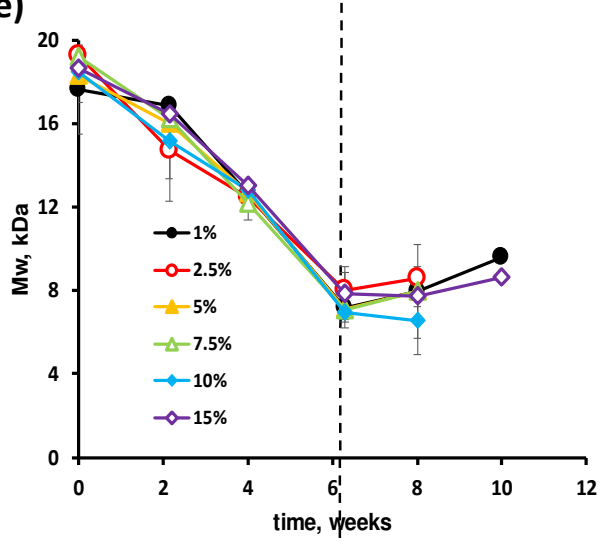

f)

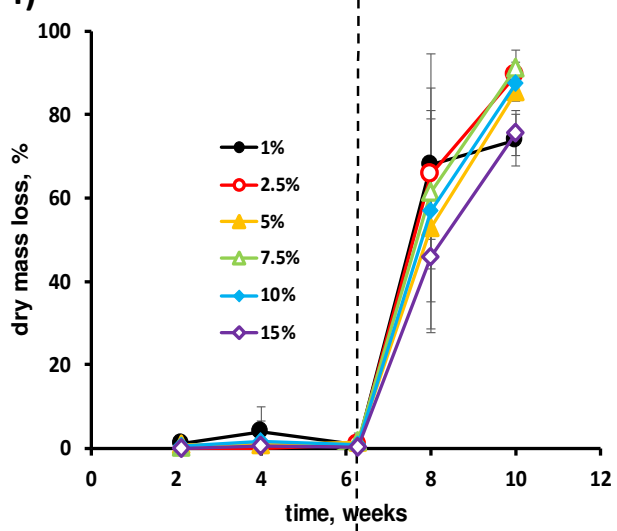

g)

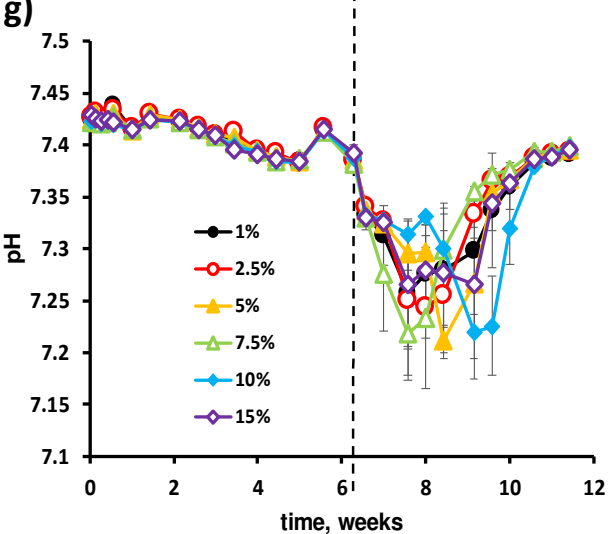




\subsection{The orchestrating role of PLGA/PLA swelling for drug release}

The hypothesized drug release mechanisms for the 3 types of PLGA/PLA-based implants are schematically illustrated in Figure 7 . They are valid for all the investigated polymers types and drug loadings.

Initially, the drug is effectively trapped within the polymeric matrix. Upon contact with aqueous fluids, limited amounts of water penetrate into the implants (the water contents of the latter does not exceed $60 \%$ ). This is because of the hydrophobicity of the PLGA/PLA chains and their intensive entanglement. The amounts of water entering the implants at this stage are not sufficient to allow for significant drug dissolution and mobility: The drug is still effectively trapped and release into the surrounding bulk fluid is negligible. However, the limited amounts of water entering the implants upon exposure to the release medium are sufficient to cause polymer degradation throughout the devices. Hence, the polymer molecular weight decreases and the PLGA/PLA chains become more hydrophilic and less entangled. Also, water-soluble degradation products build up a steadily increasing osmotic pressure, attracting water into the system. Once a critical threshold range (around $8 \mathrm{kDa}$ ) is reached, substantial polymer swelling sets on: The newly created $-\mathrm{COOH}$ end groups, lower polymer chain entanglement and generated osmotic pressure lead to water contents exceeding 80-90\% in the implants. Obviously, this represents fundamentally altered conditions for drug release: Considerable amounts of water are now available for drug dissolution and the mobility of dissolved drug molecules is dramatically increased. Due to concentration gradients, the drug diffuses out of the implants into the surrounding bulk fluid: Drug release sets on.

In brief, polymer swelling "orchestrates" drug release: It enables drug release by fundamentally changing the conditions for drug dissolution and diffusion.

As also the mobility of the generated shorter chain acids substantially increases upon PLGA/PLA swelling, they can diffuse out into the release medium, too. The loss of these compounds as well as the loss of the drug result in the onset of the dry mass loss of the implants. The time point at which polymer swelling sets on depends on the type of PLGA/PLA.

Please note that, thus, also the onset of major "dry mass loss" roughly coincides with the onset of drug release in all the investigated systems (Figures 3-6). But we do not believe that this onset of substantial dry mass loss is the root cause for the onset of drug release in these systems. We believe that the substantial implant swelling fundamentally changes the conditions for: (i) drug dissolution and diffusion, and (ii) the diffusion of water-soluble oligomers and monomers out of the implants. These crucial changes in the conditions for mass transport are believed to be the root cause for the observed onset of substantial dry mass loss (not vice-versa).

\section{Conclusion}

PLGA/PLA swelling seems to control the conditions for drug dissolution and diffusion in the investigated hot melt extruded implants: Initially, the polymers are too hydrophobic to allow for substantial water penetration into the system. Hence, the drug is not sufficiently mobile to be released. But the limited amounts of water that penetrate into the implants cleave the polyesters. Since the polymer end groups are hydrophilic (-COOH terminated), the PLGA/PLA becomes more hydrophilic over time. Once a critical threshold range around $8 \mathrm{kDa}$ is reached, substantial amounts of water come in (the water content exceeds 80-90\%). This fundamentally changes the environment of the drug: The latter can dissolve and becomes mobile in the highly swollen implants: Drug release starts.

Please note that in this study hot melt extruded implants were studied, which were loaded with 1-15\% dexamethasone, a drug with limited water-solubility. In other types of implants, additional phenomena might be of importance, e.g. pronounced osmotic effects due to the 
presence of high amounts of freely water-soluble drugs, or an initial network of pores. Also, potentially non-homogenous drug distributions within the implant might affect the resulting drug release kinetics. In the future it will be interesting to study the potential impact of the type of preparation technique and device design (e.g. composition, geometry and dimensions) on the "orchestrating" role of PLGA/PLA swelling for drug release.

\section{Acknowledgments}

The authors would like to thank Mr. A. Fadel from the "Centre Commun de Microscopie" of the University of Lille ("Plateau technique de le Federation Chevreul CNRS FR 2638") and Mr. J. Verin (INSERM U1008, University of Lille) for their valuable technical help with the SEM pictures. 

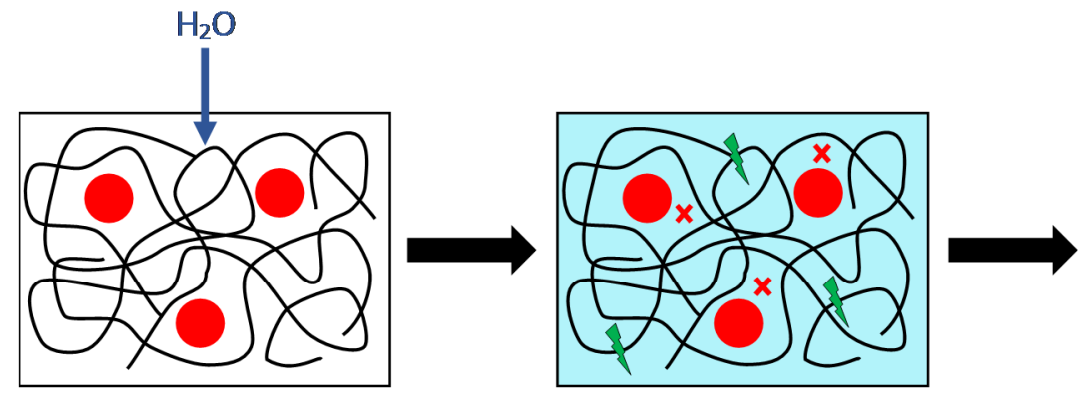

Bulk degradation

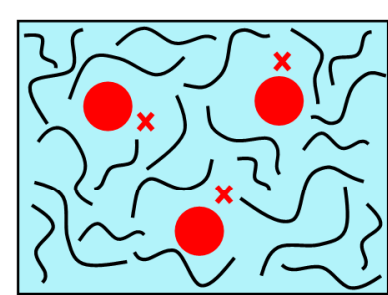

Crit. MW

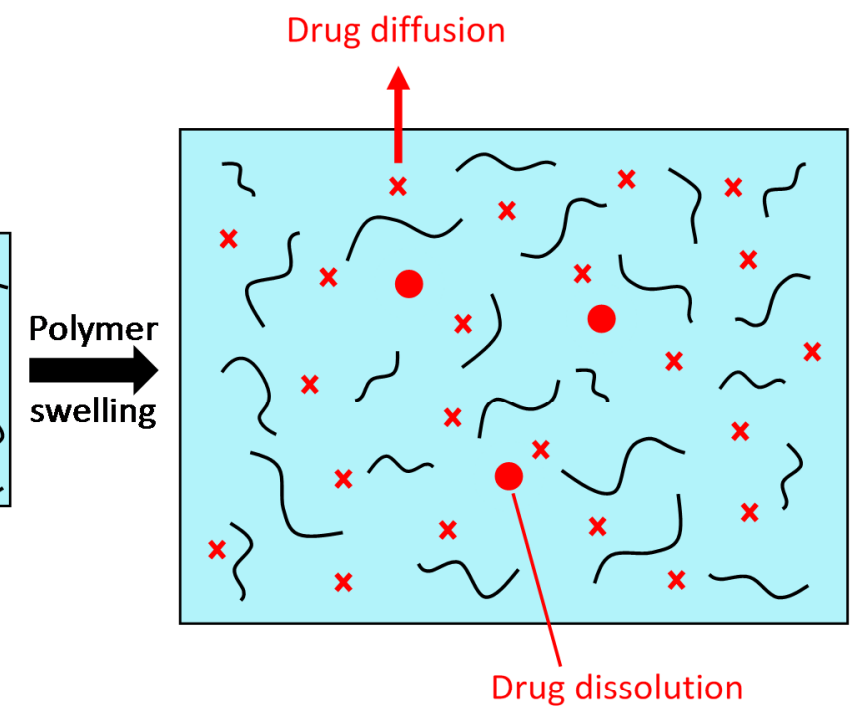

Drug particle

$\times \quad$ Drug molecule

\section{$\sim$ Polymer chain}

Figure 7: Schematic presentation of the involved mass transport phenomena involved in the control of drug release from the investigated PLGA/PLA-based implants. Polymer swelling "orchestrates" drug release: after a certain lag time, it fundamentally changes the conditions for drug dissolution and diffusion, and drug release sets 


\section{References}

[1] J. Mauduit, N. Bukh, and M. Vert, «Gentamycin/poly(lactic acid) blends aimed at sustained release local antibiotic therapy administered per-operatively. I. The case of gentamycin base and gentamycin sulfate in poly(dl-lactic acid) oligomers», $J$. Controlled Release, vol. 23, n 3, p. 209-220, 1993.

[2] C. Berkland, M. King, A. Cox, K. (Kevin) Kim, and D. W. Pack, «Precise control of PLG microsphere size provides enhanced control of drug release rate», J. Controlled Release, vol. 82, n 1, p. 137-147, 2002.

[3] K. Hirota et al., «Characterizing release mechanisms of leuprolide acetate-loaded PLGA microspheres for IVIVC development I: In vitro evaluation», J. Controlled Release, vol. 244, p. 302-313, 2016.

[4] R. B. Shah and S. P. Schwendeman, «A biomimetic approach to active selfmicroencapsulation of proteins in PLGA», J. Controlled Release, vol. 196, p. 60-70, 2014.

[5] Y. Zhang and S. P. Schwendeman, «Minimizing acylation of peptides in PLGA microspheres», J. Controlled Release, vol. 162, n 1, p. 119-126, 2012.

[6] J. M. Anderson and M. S. Shive, «Biodegradation and biocompatibility of PLA and PLGA microspheres», Adv. Drug Deliv. Rev., vol. 28, n 1, p. 5-24, 1997.

[7] M. A. Tracy et al., «Factors affecting the degradation rate of poly(lactide-co-glycolide) microspheres in vivo and in vitro», Biomaterials, vol. 20, n 11, p. 1057-1062, 1999.

[8] M. Vert, J. Mauduit, S. Li, «Biodegradation of PLA/GA polymers: increasing complexity», Biomaterials, vol. 15, n 15, p. 1209-1213, 1994.

[9] M. Vert, A. Torres, S. M. Li, S. Roussos, H. Garreau, «The complexity of the biodegradation of poly(2-hydroxy acid)-type aliphatic polyesters», in Studies in Polymer Science, vol. 12, Y. Doi et K. Fukuda, Ed. Elsevier, 1994, p. 11-23.

[10] E. Fournier, C. Passirani, C. N. Montero-Menei, J. P. Benoit, «Biocompatibility of implantable synthetic polymeric drug carriers: focus on brain biocompatibility», Biomaterials, vol. 24, $\mathrm{n}^{\mathrm{o}}$ 19, p. 3311-3331, 2003.

[11] A. Sheikh Hasan, A. Sapin, C. Damgé, P. Leroy, M. Socha, P. Maincent, «Reduction of the in vivo burst release of insulin-loaded microparticles», J. Drug Deliv. Sci. Technol., vol. 30, p. 486-493, 2015.

[12] P. Johansen, G. Corradin, H. P. Merkle, B. Gander, «Release of tetanus toxoid from adjuvants and PLGA microspheres: How experimental set-up and surface adsorption fool the pattern», J. Controlled Release, vol. 56, n 1, p. 209-217, 1998.

[13] G. Jiang, B. H. Woo, F. Kang, J. Singh, P. P. DeLuca, «Assessment of protein release kinetics, stability and protein polymer interaction of lysozyme encapsulated poly(d,1lactide-co-glycolide) microspheres», J. Controlled Release, vol. 79, nº 1, p. 137-145, 2002.

[14] H. B. Ravivarapu, K. Burton, P. P. DeLuca, «Polymer and microsphere blending to alter the release of a peptide from PLGA microspheres», Eur. J. Pharm. Biopharm., vol. 50, $n^{0} 2$, p. 263-270, 2000.

[15] S. Fischer, C. Foerg, S. Ellenberger, H. P. Merkle, B. Gander, «One-step preparation of polyelectrolyte-coated PLGA microparticles and their functionalization with model ligands», J. Controlled Release, vol. 111, nº 1, p. 135-144, 2006.

[16] M. A. Ibrahim, A. Ismail, M. I. Fetouh, A. Göpferich, «Stability of insulin during the erosion of poly(lactic acid) and poly(lactic-co-glycolic acid) microspheres», $J$. Controlled Release, vol. 106, $\mathrm{n}^{\mathrm{o}}$ 3, p. 241-252, 2005.

[17] A. R. Ahmed and R. Bodmeier, «Preparation of preformed porous PLGA microparticles and antisense oligonucleotides loading», Eur. J. Pharm. Biopharm., vol. 71, no 2, p. 264-270, 2009. 
[18] A. Giteau, M. C. Venier-Julienne, A. Aubert-Pouëssel, J. P. Benoit, «How to achieve sustained and complete protein release from PLGA-based microparticles?», Int. J. Pharmaceut., vol. 350, $\mathrm{n}^{\mathrm{o}}$ 1, p. 14-26, 2008.

[19] C. Wischke and S. P. Schwendeman, «Principles of encapsulating hydrophobic drugs in PLA/PLGA microparticles», Int. J. Pharmaceut., vol. 364, n 2, p. 298-327, 2008.

[20] S. Marquette et al., «Stability study of full-length antibody (anti-TNF alpha) loaded PLGA microspheres», Int. J. Pharmaceut., vol. 470, n 1, p. 41-50, 2014.

[21] B. Albertini et al., « $\beta$-cyclodextrin hinders PLGA plasticization during microparticle manufacturing», J. Drug Deliv. Sci. Technol., vol. 30, p. 375-383, 2015.

[22] F. Wan et al., «Impact of PLGA molecular behavior in the feed solution on the drug release kinetics of spray dried microparticles», Polymer, vol. 54, ${ }^{0}$ 21, p. 5920-5927, 2013.

[23] N. Samadi et al., «The effect of lauryl capping group on protein release and degradation of poly(d,1-lactic-co-glycolic acid) particles», J. Controlled Release, vol. 172, $\mathrm{n}^{\mathrm{o}}$ 2, $\mathrm{p}$. 436-443, 2013.

[24] X. Luan and R. Bodmeier, «Modification of the tri-phasic drug release pattern of leuprolide acetate-loaded poly(lactide-co-glycolide) microparticles», Eur. J. Pharm. Biopharm., vol. 63, $\mathrm{n}^{\mathrm{o}}$ 2, p. 205-214, 2006.

[25] F. Ramazani et al., «Formulation and characterization of microspheres loaded with imatinib for sustained delivery», Int. J. Pharmaceut., vol. 482, nº 1, p. 123-130, 2015.

[26] S. Kempe, H. Metz, K. Mäder, «Do in situ forming PLG/NMP implants behave similar in vitro and in vivo? A non-invasive and quantitative EPR investigation on the mechanisms of the implant formation process», J. Controlled Release, vol. 130, $\mathrm{n}^{\mathrm{o}} 3, \mathrm{p}$. 220-225, 2008.

[27] N. Faisant, J. Siepmann, J. P. Benoit, «PLGA-based microparticles: elucidation of mechanisms and a new, simple mathematical model quantifying drug release», Eur. $J$. Pharm. Sci., vol. 15, no 4, p. 355-366, 2002.

[28] P. Blasi, S. S. D’Souza, F. Selmin, P. P. DeLuca, «Plasticizing effect of water on poly(lactide-co-glycolide)», J. Controlled Release, vol. 108, $\mathrm{n}^{\mathrm{o}}$ 1, p. 1-9, 2005.

[29] S. Fredenberg, M. Wahlgren, M. Reslow, A. Axelsson, «The mechanisms of drug release in poly(lactic-co-glycolic acid)-based drug delivery systems-A review», Int. J. Pharmaceut., vol. 415, n ${ }^{\circ} 1-2$, p. 34-52, 2011.

[30] A. C. Doty et al., «Mechanisms of in vivo release of triamcinolone acetonide from PLGA microspheres», J. Controlled Release, vol. 256, p. 19-25, 2017.

[31] J. Siepmann and A. Göpferich, «Mathematical modeling of bioerodible, polymeric drug delivery systems», Adv. Drug Deliv. Rev., vol. 48, n 2-3, p. 229-247, 2001.

[32] M. Husmann, S. Schenderlein, M. Lück, H. Lindner, P. Kleinebudde, «Polymer erosion in PLGA microparticles produced by phase separation method», Int. J. Pharmaceut., vol. 242, $\mathrm{n}^{\mathrm{o}} 1$, p. 277-280, 2002.

[33] J. Siepmann and F. Siepmann, «Mathematical modeling of drug dissolution», Int. J. Pharmaceut., vol. 453, $\mathrm{n}^{\mathrm{o}}$ 1, p. 12-24, 2013.

[34] S. Fredenberg, M. Jönsson, T. Laakso, M. Wahlgren, M. Reslow, A. Axelsson, «Development of mass transport resistance in poly(lactide-co-glycolide) films and particles - A mechanistic study», Int. J. Pharmaceut., vol. 409, nº 1, p. 194-202, 2011.

[35] L. Wu, J. Zhang, D. Jing, J. Ding "Wet-state" mechanical properties of threedimensional polyester porous scaffolds», J. Biomed. Mater. Res. A, vol. 76A, p. 264271, 2006.

[36] S. M. Li, H. Garreau, M. Vert, «Structure-property relationships in the case of the degradation of massive aliphatic poly-( $\alpha$-hydroxy acids) in aqueous media», J. Mater. Sci. Mater. Med., vol. 1, nº 3, p. 123-130, 1990. 
[37] S. M. Li, H. Garreau, M. Vert, «Structure-property relationships in the case of the degradation of massive poly( $\alpha$-hydroxy acids) in aqueous media», J. Mater. Sci. Mater. Med., vol. 1, nº 3, p. 131-139, 1990.

[38] M. Therin, P. Christel, S. Li, H. Garreau, M. Vert, «In vivo degradation of massive poly $\left(\alpha\right.$-hydroxy acids): Validation of In vitro findings», Biomaterials, vol. 13, $\mathrm{n}^{\circ}$ 9, $\mathrm{p}$. 594-600, 1992.

[39] K. Mäder, B. Gallez, K. J. Liu, H. M. Swartz, «Non-invasive in vivo characterization of release processes in biodegradable polymers by low-frequency electron paramagnetic resonance spectroscopy», Biomaterials, vol. 17, n 4, p. 457-461, 1996.

[40] F. von Burkersroda, L. Schedl, A. Göpferich, «Why degradable polymers undergo surface erosion or bulk erosion», Biomaterials, vol. 23, n 21, p. 4221-4231, 2002.

[41] A. Brunner, K. Mäder, A. Göpferich, «pH and Osmotic Pressure Inside Biodegradable Microspheres During Erosion1», Pharm. Res., vol. 16, nº 6, p. 847-853, 1999.

[42] A. Schädlich, S. Kempe, K. Mäder, «Non-invasive in vivo characterization of microclimate $\mathrm{pH}$ inside in situ forming PLGA implants using multispectral fluorescence imaging», J. Controlled Release, vol. 179, p. 52-62, 2014.

[43] K. Fu, D. W. Pack, A. M. Klibanov, R. Langer, «Visual evidence of acidic environment within degrading poly(lactic-co-glycolic acid) (PLGA) microspheres», Pharm. Res., vol. 17, $\mathrm{n}^{\mathrm{o}}$ 1, p. 100-106, 2000.

[44] D. Klose, F. Siepmann, K. Elkharraz, S. Krenzlin, J. Siepmann, «How porosity and size affect the drug release mechanisms from PLGA-based microparticles», Int. J. Pharmaceut., vol. 314, nº 2, p. 198-206, 2006.

[45] J. Siepmann, K. Elkharraz, F. Siepmann, D. Klose, «How Autocatalysis Accelerates Drug Release from PLGA-Based Microparticles: A Quantitative Treatment», Biomacromolecules, vol. 6, no 4, p. 2312-2319, 2005.

[46] J. Wang, B. M. Wang, S. P. Schwendeman, «Characterization of the initial burst release of a model peptide from poly(d,l-lactide-co-glycolide) microspheres», J. Controlled Release, vol. 82, nº 2, p. 289-307, 2002.

[47] J. Kang and S. P. Schwendeman, «Pore closing and opening in biodegradable polymers and their effect on the controlled release of proteins», Mol. Pharm., vol. 4, $\mathrm{n}^{\mathrm{o}}$ 1, $\mathrm{p}$. 104-118, 2007.

[48] H. Gasmi, F. Danede, J. Siepmann, F. Siepmann, «Does PLGA microparticle swelling control drug release? New insight based on single particle swelling studies», $J$. Controlled Release, vol. 213, p. 120-127, 2015.

[49] H. Gasmi, J.-F. Willart, F. Danede, M. C. Hamoudi, J. Siepmann, F. Siepmann, «Importance of PLGA microparticle swelling for the control of prilocaine release», $J$. Drug Deliv. Sci. Technol., vol. 30, p. 123-132, 2015.

[50] H. Gasmi et al., «Towards a better understanding of the different release phases from PLGA microparticles: Dexamethasone-loaded systems», Int. J. Pharmaceut., vol. 514, $\mathrm{n}^{\mathrm{o}} 1$, p. 189-199, 2016.

[51] W. Friess and M. Schlapp, «Release mechanisms from gentamicin loaded poly(lacticco-glycolic acid) (PLGA) microparticles», J. Pharm. Sci., vol. 91, n 3, p. 845-855, 2002.

[52] C. Berkland, E. Pollauf, C. Raman, R. Silverman, K. (Kevin) Kim, D. W. Pack, «Macromolecule Release from Monodisperse PLG Microspheres: Control of Release Rates and Investigation of Release Mechanism», J. Pharm. Sci., vol. 96, n ${ }^{0}$ 5, p. 1176-1191, 2007.

[53] I. Mylonaki, E. Allémann, F. Delie, O. Jordan, «Imaging the porous structure in the core of degrading PLGA microparticles: The effect of molecular weight», J. Controlled Release, vol. 286, p. 231-239, 2018. 
[54] M. Beugeling et al., «The mechanism behind the biphasic pulsatile drug release from physically mixed poly(dl-lactic(-co-glycolic) acid)-based compacts», Int. J. Pharmaceut., vol. 551, no 1-2, p. 195-202, 2018.

[55] T. G. Park, «Degradation of poly(d,1-lactic acid) microspheres: effect of molecular weight», J. Controlled Release, vol. 30, nº 2, p. 161-173, 1994. 
Polymer swelling orchestrates drug release from PLGA implants
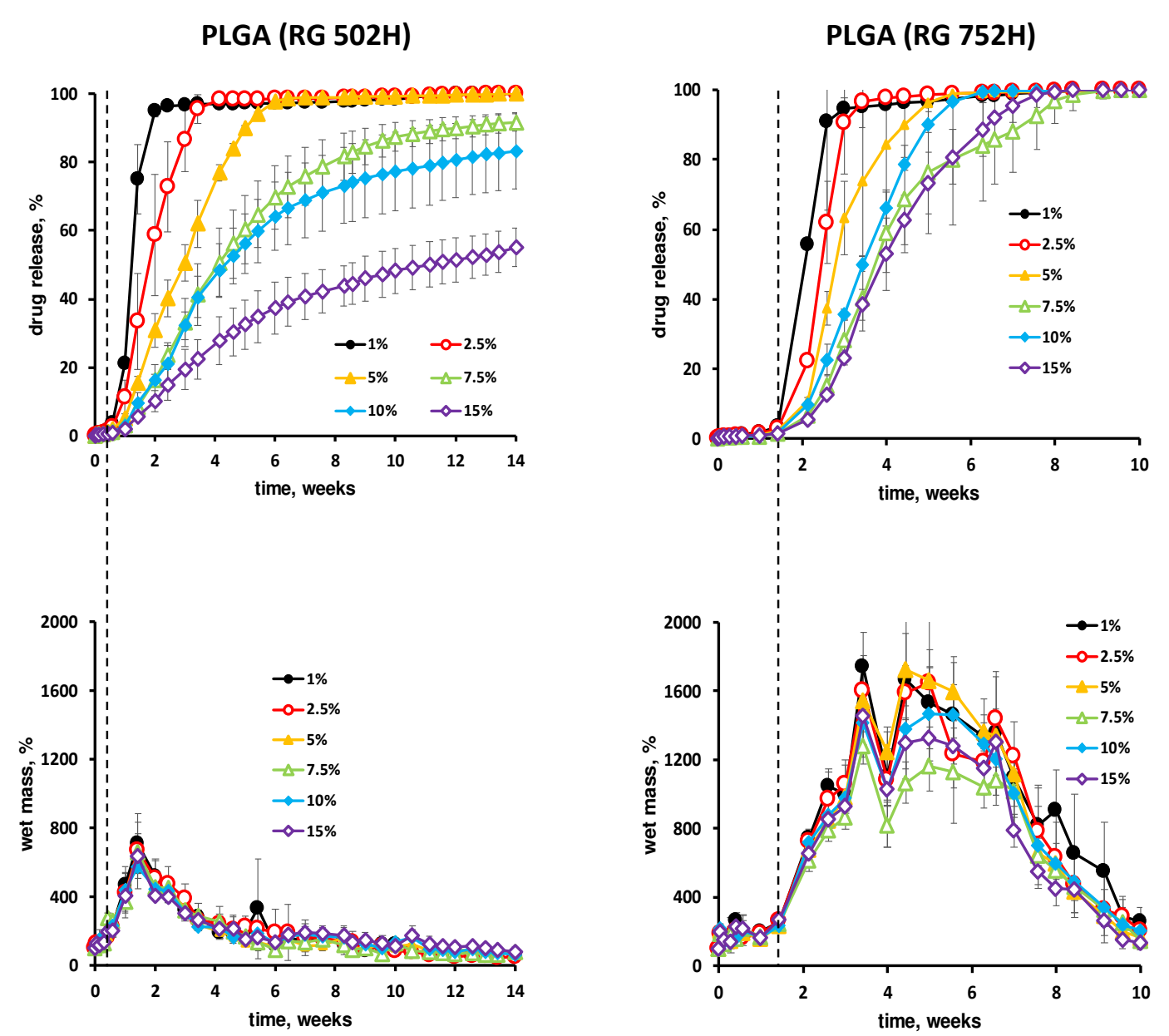

PLA (R 202H)
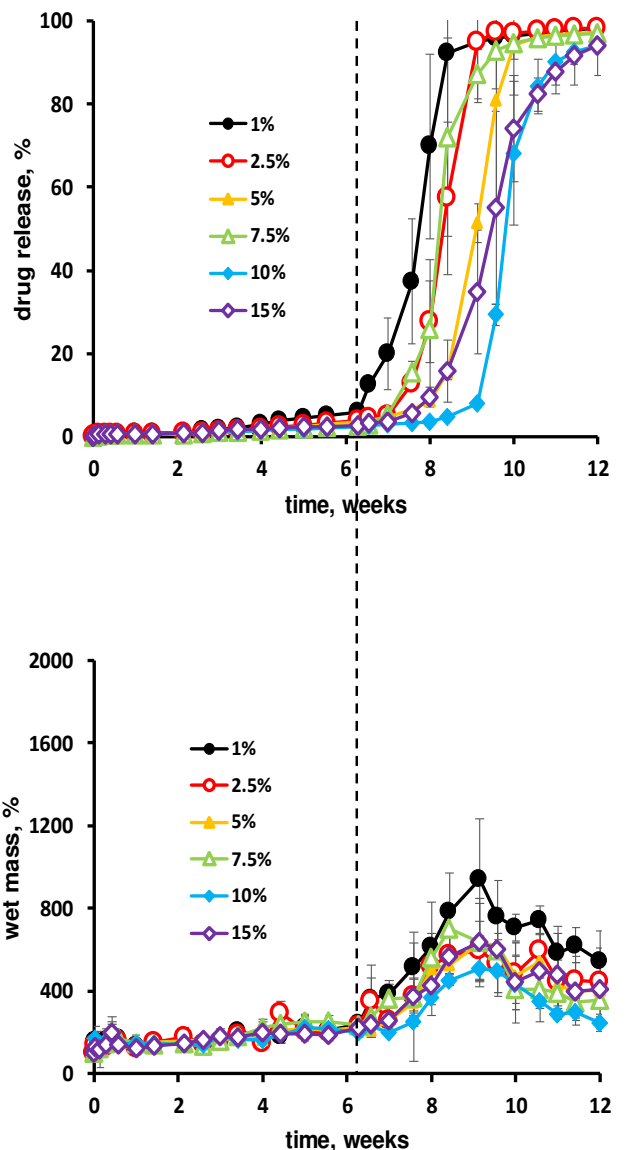